\title{
ON A TRANSLATION PROPERTY OF POSITIVE DEFINITE FUNCTIONS
}

\author{
LARS OMLOR \\ Section for Computational Sensomotorics \\ Hertie Institute for Clinical Brain Research $\&$ Center for Integrative Neuroscience \\ University of Tübingen \\ D-72076 Tübingen, Germany \\ E-mail: lars.omlor@medizin.uni-tuebingen.de \\ MICHAEL LEINERT \\ Institute of Applied Mathematics, University of Heidelberg \\ D-69120 Heidelberg, Germany \\ e-mail: leinert@math.uni-heidelberg.de
}

\begin{abstract}
If $G$ is a locally compact group with a compact invariant neighbourhood of the identity $e$, the following property $(*)$ holds: For every continuous positive definite function $h \geq 0$ with compact support there is a constant $C_{h}>0$ such that $\int L_{x} h \cdot g \leq C_{h} \int h g$ for every continuous positive definite $g \geq 0$, where $L_{x}$ is left translation by $x$. In [L], property $(*)$ was stated, but the above inequality was proved for special $h$ only. That "for one $h$ " implies "for all $h$ " seemed obvious, but turned out not to be obvious at all. We fill this gap by means of a new structure theorem for IN-groups.

For $p \in \mathbb{N}$ even, property $(*)$ easily implies the following property $(*)_{p}$ : For every relatively compact invariant neighbourhood $U$ of $e$, there is a constant $C_{U}>0$ such that $\left\|\chi_{x U} \cdot g\right\|_{p} \leq$ $C_{U}\left\|\chi_{U} \cdot g\right\|_{p}$ for every continuous positive definite function $g$. For all other $p \in(1, \infty)$, property $(*)_{p}$ fails (see $[\mathrm{L}]$ ). In the special case of the unit circle, the \|\|$_{p}$-norm results are essentially due to N. Wiener, S. Wainger, and H. S. Shapiro. For compact abelian groups they are due to M. Rains, and for locally compact abelian groups to J. Fournier.
\end{abstract}

$G$ denotes a locally compact group with identity element $e$. We assume $G$ to be an IN-group (IN = "invariant neighbourhood"), i.e. $G$ has a compact neighbourhood of $e$ which is invariant under inner automorphisms of $G$. Integrals are taken with respect

2000 Mathematics Subject Classification: 22D05, 43A35, 43-06.

Key words and phrases: IN-group, structure theorem, positive definite function. The paper is in final form and no version of it will be published elsewhere. 
to left Haar measure on $G$, and subsets of $G$ which we consider are assumed to be Haar measurable. By $P(G)$ resp. $P_{c}(G)$ we denote the set of positive definite continuous functions on $G$ resp. the subset of those $f$ in $P(G)$ whose $\operatorname{support} \operatorname{supp}(f)$ is compact. The characteristic function of a set $A \subset G$ is denoted $\chi_{A}$. Left translation of a function $f$ by $a \in G$ is defined by $L_{a} f(x)=f\left(a^{-1} x\right)$ for $x \in G$. As usual, convolution and involution of functions are written $f * g$ and $f^{*}$ respectively. Since IN-groups are unimodular, the involution is given by $f^{*}(x)=\overline{f\left(x^{-1}\right)}$ for $x \in G$. The Dirac measure in the point $a \in G$ is $\delta_{a}$. If $\pi$ is a unitary representation of $G$, we denote its integrated version on the algebra $L^{1}(G)$ of integrable functions by $\pi$ again.

Definition. We say that $G$ has property $(*)$ if for every non-negative $h \in P_{c}(G)$ there is some $C_{h}>0$ such that $\int L_{x} h \cdot g \leq C_{h} \int h g$ for all $x \in G$ and all non-negative $g \in P(G)$.

Lemma 1. Let $G$ be locally compact. Let $x \in G$ and $U$ be a relatively compact neighbourhood of e such that $\delta_{x} * \chi_{U}=\chi_{U} * \delta_{x}$. Then, letting $h=\chi_{U}^{*} * \chi_{U}$, we have

(i) $\int L_{x} h \cdot g \leq \int h \cdot g$ for all non-negative $g \in P(G)$.

In particular, (i) holds if $x$ is in the center of $G$ or $U$ is an invariant neighbourhood of e.

Proof. We may write $g$ in the form $g(x)=\langle\pi(x) \xi, \xi\rangle$ where $\pi$ is a unitary representation of $G$ and $\xi$ is a cyclic vector of $\pi$. Now

$$
\begin{aligned}
\int L_{x} h \cdot g=\left\langle\pi\left(L_{x} h\right) \xi, \xi\right\rangle=\left\langle\pi(x) \pi\left(\chi_{U}^{*}\right)\right. & \left.\pi\left(\chi_{U}\right) \xi, \xi\right\rangle=\left\langle\pi(x) \pi\left(\chi_{U}\right) \xi, \pi\left(\chi_{U}\right) \xi\right\rangle \\
\leq\left\|\pi\left(\chi_{U}\right) \xi\right\|^{2} & =\left\langle\pi\left(\chi_{U}^{*} * \chi_{U}\right) \xi, \xi\right\rangle=\int h g .
\end{aligned}
$$

REMARK. Let $f, h \in P_{c}(G)$ be non-negative and suppose there is $C_{h}>0$ such that $\int L_{x} h \cdot g \leq C_{h} \int h g$ for all $x \in G$ and non-negative $g \in P(G)$. If $f>0$ on supp $h$, a corresponding inequality $\int L_{x} f \cdot g \leq C_{f} \int f g$ holds. This follows from the fact that there are constants $c_{0}, \ldots, c_{n}>0$ and elements $x_{1}, \ldots, x_{n} \in G$ such that $h \leq c_{0} f$ and $f \leq \sum_{i=1}^{n} c_{i} L_{x_{i}} h$.

LEMma 2. Let $G$ be an IN-group and $H$ an open normal subgroup of $G$. If $H$ has property (*), so has $G$.

Proof. By the Remark, it suffices to prove inequality (i) for functions $h=\chi_{V}^{*} * \chi_{V}$ where $V$ is a small compact neighbourhood of $e$ in $G$. If $U$ is an invariant compact neighbourhood of $e$ in $G$, so is $U \cap H$. Let $f=\chi_{U \cap H}^{*} * \chi_{U \cap H}$. Let $h=\chi_{V}^{*} * \chi_{V}$ with $V \subset U \cap H$. For non-negative $g \in P(G)$ and any $x \in G$ we have $\int L_{x} h \cdot g \leq \int L_{x} f \cdot g \leq$ $\int f g$ by Lemma 1 . Since $f$ and $h$ have support in $H$, there are $y_{1}, \ldots, y_{n} \in H$ and constants $c_{1}, \ldots, c_{n}>0$ such that $f \leq \sum_{i=1}^{n} c_{i} L_{y_{i}} h$. Hence $\int f g \leq \sum_{i=1}^{n} c_{i} \int L_{y_{i}} h \cdot g \leq$ $\sum_{i=1}^{n} c_{i} C_{h}^{H} \int h g$, since these integrals are in $H$ and $H$ has property $(*)$. Altogether we have $\int L_{x} h \cdot g \leq C_{h}^{H}\left(\sum_{i=1}^{n} c_{i}\right) \int h g$, which proves property $(*)$ for $G$.

Lemma 3. Let $G$ be an IN group, $V$ an open neighbourhood of e, $\mathcal{F}$ the system of all compact invariant neighbourhoods of e, and $N=\bigcap_{U \in \mathcal{F}} U$. Then there are a compact invariant neighbourhood $M$ of e and elements $n_{1}, \ldots, n_{k} \in N$ such that $M \subset \bigcup_{j=1}^{k} n_{j} V$. 
Proof. Since $N$ is compact, there are $n_{1}, \ldots, n_{k} \in N$ with $N \subset \bigcup_{j=1}^{k} n_{j} V$. This means $\bigcap_{U \in \mathcal{F}}\left(U \backslash \bigcup_{j=1}^{k} n_{j} V\right)=\emptyset$. This being an intersection of compact sets, the finite intersection property implies that there is a finite subsystem $\mathcal{F}^{\prime} \subset \mathcal{F}$ with $\bigcap_{U \in \mathcal{F}^{\prime}}\left(U \backslash \bigcup_{j=1}^{k} n_{j} V\right)$ $=\emptyset$, that is, $\bigcap_{U \in \mathcal{F}^{\prime}} \subset \bigcup_{j=1}^{k} n_{j} V$. So take $M=\bigcap_{U \in \mathcal{F}^{\prime}} U$.

THEOREM 1. Every IN-group $G$ contains an open normal subgroup $H$ such that the intersection of all compact $H$-invariant neighbourhoods of the identity of $H$ is contained in the center of $H$.

Proof. Let $G$ be an arbitrary IN-group with $N_{G}$ the intersection of all its compact invariant neighbourhoods of the identity. The group $G / N_{G}$ is SIN and therefore-according to theorem 2.13 in GM] - an extension: $\{1\} \rightarrow V \times K \rightarrow G / N_{G} \rightarrow D \rightarrow\{1\}$, in which $D$ is discrete, $V$ a vector subgroup and $K$ compact. The inverse image of $V \times K$ under the canonical group homomorphism $G \rightarrow G / N_{G}$ is an open normal IN-subgroup $H$ of $G$ with $G / H \simeq D$

In particular $N_{H} \subset N_{G} \unlhd H$ and

$$
H / N_{G}=V \times K
$$

Let $L$ be the inverse image of $K$ under the canonical group homomorphism $H \rightarrow H / N_{G}$. Then $L$ is a compact normal subgroup of $H$ with $H / L \simeq{ }^{H} / N_{G} / L / N_{G}=V \times K / K=V$.

The component of the identity of locally compact groups is fully characteristic (Lemma 7 in $[\mathrm{H}]$ ). Thus

$$
\begin{aligned}
& V=V_{0}=(H / L)_{0}=H_{0} L / L \\
& \Rightarrow H / H_{0} L \simeq H / L / H_{0} L / L=V / V_{0}=\{e\} \Rightarrow H=H_{0} L \\
& \Rightarrow H / H_{0}=H_{0} L / H_{0} \simeq L / L \cap H_{0} \quad \text { which is compact. }
\end{aligned}
$$

The group $H$ therefore meets the requirements of Theorem XII in $[\mathrm{H}]$ which implies the following:

- $H$ contains a compact group $C$ and a solvable connected group $S$ such that:

$$
H / S \cap C=S / S \cap C \ltimes C / S \cap C .
$$

$S / S \cap C$ is a normal vector subgroup of $H / N_{H}$.

- $S \cap C$ is the intersection of all compact invariant neighbourhoods of $e$ in $H$, i.e. $S \cap C=N_{H}$.

- $H$ contains a subgroup $H_{1}$ of finite index. $H_{1}$ contains the component of the identity $H_{0}$ and $H_{1} / N_{H}$ is the centralizer of $S / N_{H}$ (proof in $[\mathrm{H}]$ page 53 ). Moreover $N_{H}$ is in the center of $H_{1}$.

Theorem 2.13 in GM] says that $H$ is $[F C]^{-}$. Thus $H / N_{H} \in[F C]^{-}$(being the continuous homomorphic image of an $[F C]^{-}$group ). Application of 3.4 in [GM] yields that the normal vector subgroup $S / N_{H}$ has to be central in $H / N_{H}$. In particular its centralizer $H_{1} / N_{H}$ is the whole group $H / N_{H}$. So $H_{1}$ is a subgroup of $H$ containing $N_{H}$ and intersecting every $N_{H}$ orbit. Therefore $H_{1}=H$ and $N_{H}$ is contained in the center of $H_{1}=H$.

REMARK. In the special case that $G$ is connected (then one has $H=G$ ), the theorem is due to Iwasawa [I]. 
THEOREM 2. Every IN-group has property $(*)$.

Proof Let $G$ be an IN-group and let $H$ be as in Theorem 1. By Lemma 2, it suffices to prove property $(*)$ for $H$. If $W$ is a fixed compact invariant neighbourhood of $e$ in $G$, $W^{\prime}=W \cap H$ is a compact invariant neighbourhood of $e$ in $H$, so $H$ is an IN-group, too. Let $N$ be the intersection of all compact $(H-)$ invariant neighbourhoods of $e$ in $H$. If $V$ is an open relatively compact neighbourhood of $e$ in $H$, then, by Lemma 3, there are a compact invariant neighbourhood $U$ of $e$ in $H$ and elements $n_{1}, \ldots, n_{k} \in N$ such that $U \subset \bigcup_{i=1}^{k} n_{i} V$. On the other hand, there are $y_{1}, \ldots, y_{\ell} \in H$ such that $V \subset \bigcup_{i=1}^{\ell} y_{i} U$. Taking into account that the $n_{i}$ and $\chi_{U}$ are central in $H$ and $L^{1}(H)$, respectively, we obtain

(i) $\chi_{U}^{*} * \chi_{U} \leq\left(\sum_{i=1}^{k} L_{n_{i}} \chi_{V}\right)^{*} *\left(\sum_{i=1}^{k} L_{n_{i}} \chi_{V}\right)=\sum_{i, j=1}^{k} L_{n_{i}^{-1} n_{j}} \chi_{V}^{*} * \chi_{V}$,

(ii) $\chi_{V}^{*} * \chi_{V} \leq\left(\sum_{i=1}^{\ell} L_{y_{i}} \chi_{U}\right)^{*} *\left(\sum_{i=1}^{\ell} L_{y_{i}} \chi_{U}\right)=\sum_{i, j=1}^{\ell} L_{y_{i}-1} y_{j} \chi_{U}^{*} * \chi_{U}$.

For $y \in H$ and non-negative $g \in P(H)$ we obtain from (ii) and (i) by Lemma 1

(iii) $\int L_{y} \chi_{V}^{*} * \chi_{V} \cdot g \leq \int \sum_{i, j=1}^{\ell} L_{y y_{i}^{-1} y_{j}} \chi_{U}^{*} * \chi_{U} \cdot g \leq \ell^{2} \int \chi_{U}^{*} * \chi_{U} \cdot g$,

(iv) $\int \chi_{U}^{*} * \chi_{U} \cdot g \leq \int \sum_{i, j=1}^{k} L_{n_{i}^{-1} n_{j}} \chi_{V}^{*} * \chi_{V} \cdot g \leq k^{2} \int \chi_{V}^{*} * \chi_{V} \cdot g$.

So $\int L_{y} \chi_{V}^{*} * \chi_{V} \cdot g \leq k^{2} \ell^{2} \int \chi_{V}^{*} * \chi_{V} \cdot g$. Considering small $V$ and using the Remark after Lemma 1 one sees that $H$ has property $(*)$.

Finally, the statement of Theorem 1.6 in $[\mathrm{L}]$ is correct:

COROLlaRY. If $G$ is an IN-group, $(*)_{p}$ holds for all even natural numbers $p$ and fails for all other $p \in(1, \infty)$.

The proof for even $p$ is an easy consequence of Theorem 2, using the fact that $|g|^{p}=$ $g^{p / 2} \cdot \bar{g}^{p / 2} \in P(G)$ for $g \in P(G)$ and that every $\chi_{U}$ can be sandwiched between two nonzero non-negative functions $h, h^{\prime} \in P_{c}(G)$. For the proof that property $(*)_{p}$ fails for all non-even $p \in(1, \infty)$, see $[\mathrm{L}]$.

\section{References}

[GM] S. Grosser and M. Moskowitz, Compactness conditions in topological groups, J. Reine Angew. Math. 246 (1971), 1-40.

$[\mathrm{H}] \quad$ K. H. Hofmann and P. S. Mostert, Splitting in topological groups. Mem. Amer. Math. Soc. 43 (1963).

I] K. Iwasawa, Topological groups with invariant compact neighborhoods of the identity, Ann. of Math. 54 (1951), 345-348.

L M. Leinert, On a theorem of Wiener, Manuscripta Math. 110 (2003), 1-12. 